\title{
A laboratory and field evaluation of Macrocyclops distinctus, Megacyclops viridis and Mesocyclops pehpeiensis as control agents of the dengue vector Aedes albopictus in a peridomestic area in Nagasaki, Japan
}

\author{
H. DIENG, M. BOOTS*, N. TUNO, Y. TSUDA and M. TAKAGI \\ Department of Vector Ecology and Environment, Institute of Tropical Medicine, Nagasaki, Japan and "Department of \\ Biological Sciences, Stirling University, Scotland, U.K.
}

\begin{abstract}
The use of the cyclopoid copepods Macrocyclops distinctus (Richard) Megacyclops viridis (Jurine) and Mesocyclops pehpeiensis Hu (Cyclopoida: Cyclopidae) as biological control agents against the dengue vector Aedes albopictus (Skuse) (Diptera: Culicidae) was evaluated. In the laboratory their predatory ability was highest against the younger instars of Ae. albopictus and none of the three copepods killed the fourth instar. Except for M. viridis, predatory ability was affected by the size of the container: the smaller the container, the higher the predation. A 4-month field test was conducted to examine the impact of these predators on wild Ae. albopictus. Thirty artificial containers were placed in a peridomestic area to allow Ae. albopictus colonization. We showed continuous and similar oviposition responses in treated and control containers. The densities of Ae. albopictus showed considerable short-term changes and were much reduced by the copepod species. Macrocyclops and the mixture of all three provided better Ae. albopictus control than either Megacyclops or Mesocyclops alone. When larval densities peaked in the control containers in August and September, the overall reduction due to the copepods was nearly complete. Mesocyclops inoculated alone had the highest population survival. However, the growth and survival of all the copepod species was poor when the three genera were mixed. Based on their performance and survival in the trial, Macrocyclops and Mesocyclops merit consideration as bio-control agents of Ae. albopictus.
\end{abstract}

Key words. Aedes albopictus, Macrocyclops distinctus, Megacyclops viridis, Mesocyclops pehpeiensis, growth, immature, mixture, oviposition, peridomestic area, reduction, Japan.

\section{Introduction}

Recently, copepods have emerged as a new tool for controlling mosquito vectors, particularly those inhabiting artificial containers. In Tahiti, Mesocyclops leuckarti (Claus)

Correspondence: H. Dieng, Institute of Tropical Medicine, Nagasaki University, Department of Vector Ecology and Environment, 1-12-4 Sakamoto, 852-8523 Nagasaki, Japan. E-mail: hamadydieng@hotmail.com and Mesocyclops aspericornis (Daday) were successfully used against the dengue vector Aedes aegypti (L.) (Riviere \& Thirel, 1981; Lardeaux et al., 1992) and in Honduras this mosquito was suppressed in peridomestic containers by Mesocyclops longisetus (Thiebaud) (Marten etal., 1994). In New Orleans, Macrocyclops albidus (Jurine) eliminated Aedes albopictus (Skuse) from tyre piles in a wooded area (Marten, 1990a, 1990b). More recently, in Vietnam, Mesocyclops sp. succeeded in eliminating Ae. aegypti (Nam etal., 1998) from a number of villages. This is a major advance in the biological control of disease vectors. 